\section{Desafíos en el manejo de la sedación, analgesia y bloqueo neuromuscular en el paciente crítico COVID-19 en Chile}

\author{
DANIEL MUÑOZ-PICHUANTE ${ }^{1,2, a}, J_{U A N}$ GRANDJEAN ${ }^{2,3}$, \\ FELIPE OLIVARES ${ }^{2}$, PATRICIO LEÓN ${ }^{2}$, IGNACIA ÁLVAREZ ${ }^{1, b}$
}

\section{Challenges in the management of analgesia and sedation in critically ill patients with COVID-19 in Chile}

Approximately 5\% of COVID-19 patients will have a severe disease requiring invasive or non-invasive mechanical ventilation. In this conditions, sedatives and analgesics are fundamental to promote tolerance, comfort and synchrony with the mechanical ventilator. High and unusual requirements for sedation, analgesics and neuromuscular blockers have been reported in these patients, contributing to prolonged exposure, a high rate of delirium and prolongation of mechanical ventilation. These factors, added to the progressive shortage of these drugs, a high demand for care and less capacity for personalized attention, have created an adverse scenario for their proper and rational use. This paper proposes different pharmacotherapeutic optimization strategies for a rational management of sedation, analgesia and neuromuscular block in critically ill patients with COVID-19, with the therapeutic alternatives available in Chile.

(Rev Med Chile 2021; 149: 559-569)

Key words: Coronavirus Infections; Critical Care; Deep Sedation; Analgesia; Neuromuscular Blockade.
'Instituto de Farmacia, Facultad de Ciencias, Universidad Austral de Chile.

Unidad de Cuidados Intensivos Adultos, Hospital Base Valdivia. ${ }^{3}$ Instituto de Medicina, Facultad de Medicina, Universidad Austral de Chile.

aFarmacéutico Clínico, (BCCCP). bestudiante Química y Farmacia.

Trabajo no recibió

financiamiento.

Los autores declaran no tener

conflictos de interés: ninguno.

Recibido el 22 de junio de 2020, aceptado el 3 de noviembre de 2020.

Correspondencia a:

Daniel Muñoz Pichuante Químico-Farmacéutico Instituto de Farmacia, Universidad Austral de Chile y Unidad de Cuidados Intensivos, Hospital Base Valdivia. Bueras 1003, Valdivia, Región de Los Ríos, Chile.

daniel.munoz01@uach.cl
L a enfermedad por coronavirus 2019 (COVID-19) es una condición globalmente emergente causada por el agente infeccioso SARS-CoV-2 ${ }^{1}$, el que debido a su rápida expansión ha afectado a más de 8,5 millones de personas en el mundo, de las cuales aproximadamente medio millón han fallecido ${ }^{2}$. En Chile, al momento de escribir este artículo se han confirmado 179.436 casos de COVID-19 por laboratorio, con 3.362 personas fallecidas, equivalente a una tasa de letalidad de $1,87 \%{ }^{3}$. Sumado a ello, la Región Metropolitana, la más afectada en el desarrollo de esta pandemia, reporta 1.276 pacientes en Unidades de Cuidado Intensivo (UCI), acumulando 73,71\% del total nacional, con un porcentaje de ocupación de camas críticas mayor a $90 \%{ }^{3}$.

La mayoría de los pacientes que contraen
COVID-19 cursarán con una condición leve (80\%), mientras que $20 \%$ restantes requerirán hospitalización, y aproximadamente la mitad de ellos necesitará de una Unidad de Cuidados Intensivos (UCI). De estos últimos, cerca de 90\% requerirá de intubación orotraqueal (IOT) precoz y ventilación mecánica invasiva (VMI), mientras que otro porcentaje podrá ser manejado con técnicas de ventilación mecánica no invasiva (VMNI) u oxigenoterapias de alto flujo ${ }^{4,5}$. Diferentes estudios han revelado que estos pacientes pueden permanecer largos períodos en ventilación mecánica $(>14$ días), con un grupo importante evolucionando hacia un Síndrome de Distrés respiratorio (SDRA) e hipoxemia refractaria ${ }^{6,7}$. Es esperable que, independiente la estrategia ventilatoria, estos pacientes requieran de analgésicos, sedantes o bloqueadores 
neuromusculares (BNM), con el objetivo de aliviar el dolor, mejorar la comodidad, disminuir la ansiedad y evitar asincronías ventilatorias.

Durante la pandemia hemos presenciado que la enfermedad grave o crítica por SARS-CoV-2 y sus requerimientos de atención de salud especializada, son las responsables del colapso de los sistemas hospitalarios en los distintos países, ante la necesidad masiva de camas de hospitalización y UCI y el consiguiente desabastecimiento de insumos y fármacos críticos, de ventiladores para soporte respiratorio y falta personal sanitario. Asociado, diversos factores tales como la excesiva demanda y estrés asistencial, el desgaste de los equipos de salud y el temor al contagio por extubación accidental podría generar bruscas variaciones en la calidad de la atención y seguimiento de los pacientes, impactando principalmente en terapias que requieran titulación al lado de la cama del paciente o "bedside" como es el caso de analgésicos, sedantes, bloqueantes neuromusculares o vasoactivos, aumentando el riesgo errores de medicación y otros eventos adversos graves. En este sentido, la incorporación del farmacéutico como miembro del equipo multidisciplinario en UCI y sus intervenciones "bedside", así como el desarrollo de estrategias de optimización y protocolización farmacoterapéuticas, han demostrado ser medidas efectivas en disminuir los errores de medicación, el consumo de fármacos, sus costos asociados, los días de VMI, la estadía y mortalidad en UCI, ocupando los analgésicos y sedantes un lugar prioritario dentro de los grupos terapéuticos intervenidos ${ }^{8,9}$. El objetivo del presente trabajo es proveer un revisión no sistemática, basada en la evidencia disponible de sedación, analgesia y bloqueo neuromuscular en pacientes de UCI, como estrategia para el manejo óptimo de pacientes COVID-19 graves que requieren ingreso a unidades críticas, con un énfasis en el uso racional de las alternativas terapéuticas disponibles.

\section{Oxigenoterapia y soporte ventilatorio en el paciente COVID-19}

La oxigenoterapia es parte fundamental del tratamiento de soporte para los pacientes con COVID-19 y falla ventilatoria. Las guías de la Universidad de Wuhan ${ }^{10}$, la campaña Sobreviviendo a la Sepsis ${ }^{11}$, y la guía de la Organización Panamericana de la Salud (OPS) ${ }^{12}$, recomiendan la administración de oxigenoterapia para mantener una saturación periférica de oxígeno $\left(\mathrm{SpO}_{2}\right)$ por sobre $94 \%$. Para este fin, la oxigenoterapia convencional permite un aumento progresivo en los flujos, pudiendo llegar hasta 15 litros/minuto cuando se utilizan mascarillas de recirculación. En los últimos años, se han agregado también las "cánulas nasales de alto flujo" (CNAF), las cuales suelen ser mejor toleradas que las interfaces de la VMNI, permitiendo además la administración de flujos hasta 60 litros/minuto y una fracción inspirada de $\mathrm{O}_{2}$ de hasta $100 \%$, sin impacto clínicamente relevante en la exposición a aerosoles por parte del personal de salud. Es gracias a estas características, que las CNAF se han convertido en una de las alternativas predilectas de soporte ventilatorio precoz en pacientes COVID-19, con el propósito de evitar la intubación orotraqueal (IOT), relacionada con mayores cifras de mortalidad, de complicaciones intrahospitalarias como infecciones, y otros desenlaces adversos ${ }^{12}$. Además, la ventilación en decúbito prono en pacientes con falla respiratoria hipoxémica se ha asociado a mejorías en la oxigenación, tanto en pacientes vigiles no-intubados, como en pacientes sedados profundamente e intubados, convirtiéndose en una de opción bastante atractiva en pacientes COVID-19 ${ }^{13-15}$.

Independiente de la estrategia a utilizar para el manejo de la falla respiratoria hipoxémica (catastrófica), a menudo el paciente requerirá de sedación, analgesia o bloqueo neuromuscular para disminuir el dolor asociado a los procedimientos, mejorar su acoplamiento al ventilador, disminuir la ansiedad y evitar las disincronías ventilatorias.

\section{Consideraciones generales en la sedación, analgesia y bloqueo neuromuscular de pacientes con COVID-19}

A menudo el paciente COVID-19 requerirá de ventilación mecánica prolongada y en el peor de los escenarios, esta irá acompañada de sedación profunda y requerimientos de bloqueo neuromuscular (BNM) continuo. En este contexto, cada una de estas estrategias constituirán un factor de riesgo independiente de mal pronóstico en pacientes críticos (estadía en UCI, estadía hospitalaria, días de VMI, delirium y mortalidad $)^{16,17,18}$. Sumado a ello, la atención al lado de la cama del paciente o "bedside" por parte del equipo clínico suelen ser menos frecuentes en un paciente COVID-19, ante 
la necesidad de una menor exposición del personal de salud al virus y la mayor demanda asistencial, condicionando una menor evaluación del dolor, agitación y las terapias asociadas, incrementando así el uso de analgésicos, sedantes y bloqueadores neuromusculares por fuera de los objetivos terapéuticos.

En nuestra experiencia y en la descrita por otros centros, una cantidad inusualmente elevada de pacientes ha requerido altas dosis de sedación durante el manejo de la enfermedad por SARS$\mathrm{CoV}-2$, favoreciendo la escasez de sedantes de primera línea y con ello el uso de alternativas más costosas y desconocidas. Esto, sumado a la combinación de múltiples fármacos (analgésicos, sedantes, antipsicóticos, anticonvulsivantes, entre otros) y sus diferentes mecanismos de acción, ha generado un escenario desafiante, con el potencial riesgo de efectos adversos y por ende mayor necesidad de monitoreo por el equipo clínico ${ }^{19}$.

Por otra parte, aquel paciente que se encuentre en ventilación no invasiva y en donde se pretenda evitar la IOT, será necesario optimizar la tolerancia al modo de ventilación y/o al decúbito prono, según sea al caso, lo cual estará dado por la capacidad de favorecer la comodidad y disminuir la ansiedad y activación simpática, evitando una mayor taquipnea, disnea y falla respiratoria. En este escenario, dexmedetomidina y clonidina, mediante su acción farmacológica agonista de receptores adrenérgicos alfa-2 central, serían opciones más atractivas, dado la posibilidad de ser titulados para una sedación ligera, disminuyendo la actividad simpática, sin deprimir el centro respiratorio, ofreciendo además efecto analgésico y ansiolítico $^{20,21}$.

\section{Delirium en el paciente COVID-19}

Los pacientes con COVID-19 corren el riesgo de una variedad de problemas neurológicos, especialmente en los casos más graves. Al menos 65\% de ellos cursará con delirium durante su estancia en $\mathrm{UCI}^{22}$, favorecido además por otros factores, como dolor, infección, aporte nutricional, constipación, hidratación e hipoxia, medicamentos y metabolismo, y ambientales, los que pueden fácilmente recordarse con la mnemotecnia "PINCH-ME" (por sus siglas en inglés Pain, Infection, Nutrition, Constipation, Hydration and Hypoxia, Medication and Metabolic, Enviroment) ${ }^{23}$. Reportes recientes en pacientes con COVID-19, han documentado que el delirium puede ser producto de una invasión directa del virus al sistema nervioso central (SNC), la inducción de mediadores inflamatorios, así como también del desarrollo de daño hipóxico-cerebral ${ }^{24}$. Independiente del mecanismo, la presencia de delirium está asociado con mayor tiempo de VMI, estadía prolongada en UCI y hospitalaria, mayor mortalidad y peores desenlaces cognitivos post- $\mathrm{UCI}^{25}$. De este modo, el delirium corresponde a una importante complicación de la infección por SARS-CoV-2, lo que requiere poner especial esfuerzos en su diagnóstico precoz y prevención. De allí, la necesidad del seguimiento con herramientas validadas como el test CAM-ICU, en forma sistemática al menos 2 veces al día, dado el carácter fluctuante de esta afección mental ${ }^{26}$.

\section{Abordaje farmacoterapéutico óptimo de sedación, analgesia y BNM en paciente UCI}

La Sociedad Médica de Cuidados Críticos de Estados Unidos (SCCM, por sus siglas en inglés de Society of Critical Care Medicine) y la Sociedad Chilena de Medicina Intensiva, a través de sus guías para el manejo del dolor, sedación y agitación en UCI publicadas el año $2018^{27}$ y $2019^{28}$, respectivamente, han definido el manejo $\mathrm{A}, \mathrm{B}, \mathrm{C}$, D, E, F como el "gold standard" para mejorar los desenlaces asociados a sedación y analgesia en UCI. En términos simples, el " $A B C D E F$ " busca optimizar el manejo del dolor, evitar la sedación profunda, reducir el delirio, acortar la duración de la ventilación mecánica, minimizar la debilidad adquirida en UCI y fomentar la participación del paciente y la familia en los procesos de atención en esta unidad.

Parte central de esta estrategia corresponde a: 1) utilizar, de forma sistemática, escalas que evalúan dolor en pacientes críticos no comunicativos, basadas en indicadores de comportamiento, tales como BPS (Behavioral Pain Scale) y CPOT (Critical Care Pain Observation Tool), la sedación, como RASS (Richmond Agitation-Sedation Scale) y SAS (Sedation-Agitation Scale) y el delirium mediante CAM-ICU (Confusion Assessment Method in the Intensive Care Unit; 2) utilizar una sedación basada en analgésicos, tratar el dolor de forma preventiva; 3 ) favorecer una sedación ligera, priorizando el uso de dexmedetomidina y propofol; 4) evitar el uso de benzodiacepinas; 5) usar 
sedantes cuando sea estrictamente necesario y a la mínima dosis y 6) solo usar una sedación profunda cuando esté clínicamente justificado, en escenarios tales como SDRA grave, pacientes con necesidad de BNM, pacientes neuro-críticos con presión intra-craneana elevada, status-epilepticus refractario, hipertensión intra-abdominal, entre otros.

Aunque múltiples estudios han demostrado que llevar a cabo este "paquete de medidas" en pacientes UCI se asocia a mejores resultados clínicos, económicos y humanísticos ${ }^{29}$, su aplicación en pacientes COVID-19 debe tener presente algunas particularidades para aplicarlo de forma exitosa, las que se resumen en la Tabla 1.

De la propuesta, es relevante destacar que debe ser un objetivo prioritario el favorecer, en la medida que la condición clínica lo permita, una sedación superficial, entendida como un puntaje SAS 3 a 4 o RASS -2 a 0 , así como proactivamente titular la sedación y analgesia del paciente para conseguir sistemáticamente la administración de la mínima dosis necesaria, reevaluándose al menos cada 6 h. En este sentido, una forma ordenada, proactiva y coherente de llevarlo a cabo es "emparejando" la disminución de los requerimientos de oxígeno, tales como la fracción inspirada de oxígeno $\left(\mathrm{FiO}_{2}\right)$ y/o la presión al final de la espiración ("PEEP”), con la sedoanalgesia, bajo la premisa que, si la inestabilidad ventilatoria condiciona una mayor necesidad de sedantes y analgésicos, la estabilidad debe promover reducciones progresivas en su dosificación, evitando la acumulación de los fármacos y con ello la sobresedación. Por otra parte, un signo de alerta de dosis excesiva de analgésicos, sedantes y/o bloqueantes neuromusculares, es la ausencia de episodios agudos de agitación, dolor o disincronías ventilatorias entre evaluaciones (por ejemplo, en un intervalo de 6 h). En condiciones normales, el paciente suele tener dolor basal, sobre el cual se superponen episodios intermitentes de dolor y agitación producto de procedimientos dolorosos, incomodidad u otros. La ausencia de estos eventos agudos, evidentemente manifiestan una exposición a analgésicos y sedantes mucho mayor a las necesidades reales del paciente, condicionado en gran parte por la acumulación de ellos. Esto mismo opera para los BNM, donde la ausencia de disincronías ventilatorias intermitentes y requerimientos de bolus de BNM, deben constituir una señal de alerta para disminuir las dosis en infusión continua (Figura 1).

\section{Optimización de sedación y analgesia en el paciente crítico COVID-19}

El enfrentamiento a la sedación y analgesia en el paciente COVID-19 ha significado un escenario desafiante. Para enfrentarlo con éxito, es necesario integrar todas aquellas propuestas que han demostrado resolver de mejor manera la sedación y analgesia del paciente UCI, adaptándolas a las circunstancias actuales propias de la atención del paciente COVID-19. En este sentido, la protocolización de las actividades permitirá disminuir los tiempos de atención, disminuir los errores de medicación, utilizar de forma más eficiente los recursos y potencialmente favorecer una salida más precoz del ventilador, mejorando así el desenlace del paciente ${ }^{29}$. Acorde a los conceptos discutidos previamente, al conocimiento disponible en el manejo de pacientes UCI no-COVID-19 y a la experiencia sumada y directrices recientemente reportadas en pacientes COVID- $19^{30}$, una propuesta razonable paso a paso para optimizar el uso de la sedo-analgesia y el bloqueo neuromuscular en el paciente COVID-19, se resumen en la Figura 2.

Resulta importante destacar que la capacidad que tiene el coronavirus de invadir los nervios periféricos, descrito en modelos animales con coronavirus distintos al SARS-CoV-2, puede condicionar con mayor frecuencia y precocidad el desarrollo de neuropatías periféricas y con ello el requerimiento de terapias enfocadas en el manejo de este mecanismo doloroso, como pregabalina y carbamazepina ${ }^{22,24}$. Asociado, el uso prevalente de corticoides y bloqueadores neuromusculares, además de la inmovilidad prolongada en pacientes COVID-19, se debe considerar la neuropatía del paciente crítico como una importante variable del manejo del dolor, pudiendo contribuir a la agitación en UCI si no es reconocido y tratado en forma adecuada.

Por otra parte, el uso prolongado y/o a dosis altas de sedantes y opiáceos constituye un factor de riesgo para el desarrollo de dependencia física a estos fármacos, lo que puede ocurrir incluso antes de los 7 días en pacientes $\mathrm{UCI}^{31}$. La posibilidad de que se manifieste este "síndrome de abstinencia o retirada", afecta potencialmente la recuperación y estadía en $\mathrm{UCI}^{32}$, pudiendo ser reducida mediante el uso de opiáceos y sedantes de acción y vida media prolongada, como metadona y diazepam, respectivamente, permitiendo 
la titulación a la baja de las infusiones intravenosas de fármacos con vida media corta, como fentanilo y midazolam.

Finalmente, las guías de sedación, analgesia y delirium de la SCCM sugieren un abordaje multimodal del dolor en pacientes UCI, entendido como enfrentamiento a diversos mecanismos y vías de acción ${ }^{28}$ a pesar de ello, la utilidad de los AINEs se ve poco recomendable dado el efecto deletéreo sobre la síntesis de prostaglandinas y el potencial impacto negativo en los trastornos de coagulación y falla renal descritas en los pacientes COVID-19, por lo que se recomienda favorecer el uso de paracetamol ${ }^{33}$.

\section{Tabla 1. Estrategias Optimización Sedo-Analgesia y BNM en pacientes críticos COVID-19}

\begin{tabular}{|c|c|}
\hline Enfoque & Recomendaciones \\
\hline Analgesia & $\begin{array}{l}\text { a. Evaluar el dolor sistemáticamente al menos cada } 6 \text { horas con una herramienta validada (BPS o CPOT). } \\
\text { b. Asumir y tratar presuntivamente el dolor en ausencia de valoración sistemática o cuando no sea posible } \\
\text { (ej: paciente con BNM). } \\
\text { c. Considerar dolor neuropático como prevalente producto de la invasión viral a los nervios periféricos y/o la } \\
\text { inmovilidad, pudiendo requerir de pregabalina. } \\
\text { d. Ante un evento agudo de dolor, preferir bolus por sobre mayor dosis en infusión. } \\
\text { e. Infusiones prolongadas y en altas dosis de opiáceos condicionarán un riesgo elevado de deprivación, } \\
\text { haciendo necesario manejarlo con estrategias terapéuticas como metadona o clonidina. } \\
\text { f. Añadir laxantes preventivamente por protocolo en todos los pacientes que inician infusión de opiáceos. } \\
\text { g. Si su paciente no requirió bolos en las } 6 \text { h previas, reducir la dosis de la infusión. } \\
\text { h. Reducir la dosis de analgésicos previo a cada Prueba de Ventilación Espontánea (PVE); realizar prueba de } \\
\text { despertar espontáneo previo a PVE (considere suspender opiáceos en infusión). } \\
\text { i. Los AINEs deben ser evitados dados sus efectos deletéreos en la síntesis de prostaglandinas y la alta preva- } \\
\text { lencia de coagulopatía y falla renal en estos pacientes. Preferir el uso de metamizol si hay fiebre persistente. }\end{array}$ \\
\hline Sedación & $\begin{array}{l}\text { a. Evaluar la sedación sistemáticamente al menos cada } 6 \text { h con una herramienta validada (SAS o RASS). } \\
\text { b. La inestabilidad hemodinámica es poco frecuente en pacientes COVID-19, haciéndolos candidatos ideales } \\
\text { para el uso de propofol o dexmedetomidina. } \\
\text { c. En pacientes en VMNI o CNAF, se sugiere utilizar dexmedetomidina o clonidina para disminuir la ansiedad } \\
\text { y activación simpática, que pueden terminar aumentando la taquipnea, disnea y necesidad de intubación } \\
\text { orotraqueal. } \\
\text { d. Ante asincronías ventilatorias, evaluar respuesta tras optimizar los parámetros del ventilador mecánico, } \\
\text { antes de incrementar la dosis de sedación. } \\
\text { e. Ante un cambio agudo en agitación, preferir el bolo por sobre mayor dosis en infusión } \\
\text { f. Infusiones prolongadas y en altas dosis de sedantes condicionarán un riesgo elevado de deprivación en } \\
\text { los pacientes, haciendo necesario manejarlos con estrategias terapéuticas como diazepam o metadona } \\
\text { (drogas de mayor vida media) } \\
\text { g. Considerar sedación profunda si el paciente cursa con SDRA y/o si requiere prono, aun cuando no todos } \\
\text { los pacientes con SDRA se beneficiarán de la sedación profunda. } \\
\text { h. Si su paciente no requirió bolos en las } 6 \text { h previas } \rightarrow \text { reducir la dosis de la infusión. } \\
\text { i. Considere reducir la dosis de sedante cada vez que el paciente disminuya sus requerimientos de oxígeno } \\
\text { (coordinar disminución FiO }{ }_{2} \text { con sedación). } \\
\text { j. Reducir la dosis de sedantes previo a cada Prueba de Ventilación Espontánea (PVE); realizar prueba de } \\
\text { despertar espontáneo previo a PVE (considere suspender sedantes en infusión). }\end{array}$ \\
\hline BNM & $\begin{array}{l}\text { a. Preferir el uso de bolus intermitentes de BNM por sobre el uso de infusiones continuas. No iniciar infusión } \\
\text { continua sin antes valorar la respuesta a dosis intermitentes. } \\
\text { b. Usar la mínima dosis necesaria de BNM para evitar disincronías con el ventilador. } \\
\text { c. Considere suspender o reducir la dosis ante ausencia de disincronías y/o requerimientos de bolos adicio- } \\
\text { nales de BNM }\end{array}$ \\
\hline
\end{tabular}

BNM: bloqueador neuromuscular; BPS: Behavioral Pain Scale; CPOT: Critical-Care Pain Observation Tool; AINEs: Antiinflamatorios no esteroidales; VMNI: Ventilación Mecánica no-Invasiva; CNAF: Cánula Nasal de Alto Flujo; Síndrome Distrés Respiratorio Agudo; $\mathrm{FiO}_{2}$ : Fracción inspirada de oxígeno. 


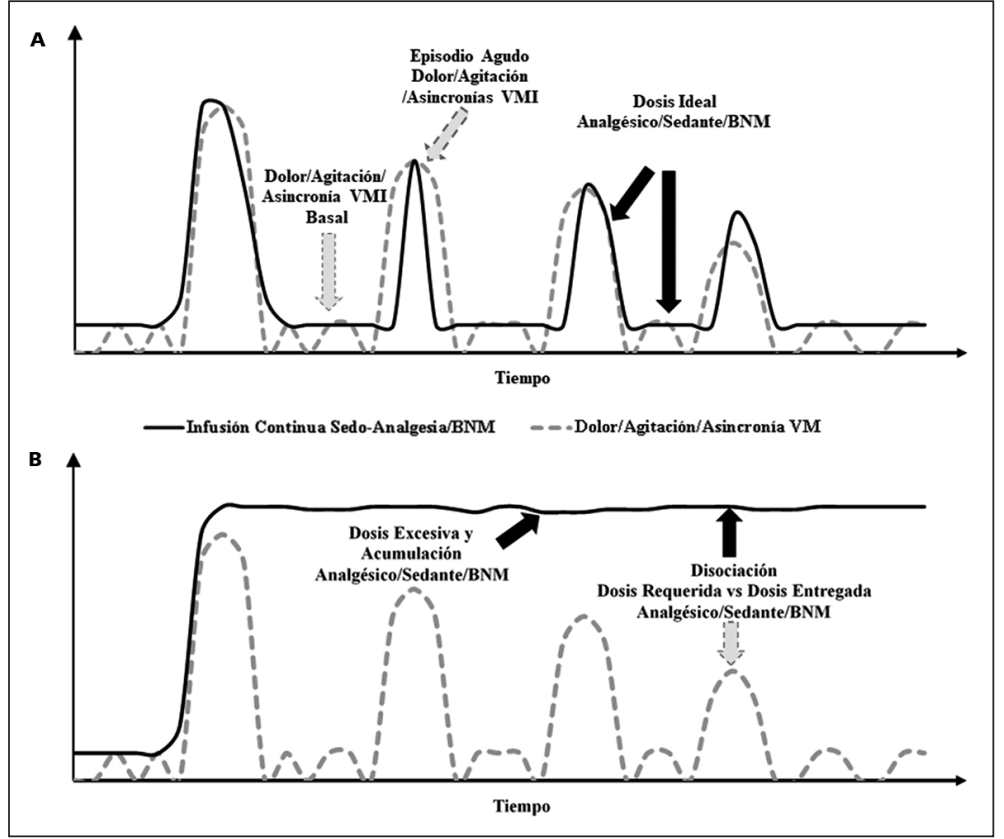

Figura 1. Relación entre los requerimientos basales de sedación, analgesia y BNM y las dosis administradas en infusión continua. La figura $\mathbf{A}$ ) retrata una situación ideal, donde los fármacos analgésicos, sedantes o BNM son titulados continuamente y se administran acorde a las necesidades basales y agudas del paciente. La figura $\mathbf{B}$ ) refleja lo que ocurre ante la ausencia de titulación de la dosis del fármaco, generando una dosis excesiva y acumulación, evidenciado por la ausencia de requerimientos agudos de analgésicos, sedantes o BNM, ante la disociación entre la dosis requerida y la entregada; BNM: bloqueo neuromuscular; VMI: ventilación mecánica invasiva.

\section{Tecnología para el monitoreo y optimización de la sedación, analgesia y BNM}

Existe en la actualidad una serie de dispositivos que permiten monitorear la profundidad de la sedación y el BNM en pacientes en VMI. Aunque estos dispositivos, tales como el Índice Biespectral (BIS) para la sedación y el Tren de Cuatro (TOF) para el BNM son recomendados en diferentes guías clínicas ${ }^{27}$, su utilidad se limita a información complementaria a la evaluación clínica y pueden no estar disponibles en todos los centros en situación de crisis. Sumado a ello, los estudios que han demostrado la utilidad de la infusión continua de BNM en SDRA, como el ACURASYS ${ }^{34}$ y ROSE ${ }^{35}$, no utilizaron TOF para definir la dosis de BNM ni tampoco BIS para evaluar la profundidad de la sedación. Cuando se decida no utilizar estos dispositivos, es importante conseguir una sedación profunda previo al inicio del BNM, la cual no podrá ser modificada hasta la suspensión del BNM. Esta estrategia de sedación comparada a su titulación utilizando BIS, no ha resultado en una diferencia significativa en la exposición de los pacientes a sedantes o analgésicos, así como en los resultados clínicos en SDRA recibiendo BNM, a pesar de la mayor cantidad de ajustes de dosis durante las primeras $24 \mathrm{~h}^{36}$. Sin embargo, es importante considerar que los estudios de NMBA en SDRA solo utilizaron BNM durante $48 \mathrm{~h}$, por lo que pueden ser necesarios monitores como BIS o TOF cuando se decida utilizar estas estrategias por $>48 \mathrm{~h}$.

\section{Propuestas de sedo-analgesia en situaciones de escasez de opciones de primera línea}

La pandemia por COVID-19 ha impactado en distintos problemas organizacionales, tales como falta de ventiladores, escasez de equipos de protección personal (EPP), recursos humanos limitados y desabastecimiento de medicamentos críticos como analgésicos y sedantes de primera y segunda línea. Ante una eventual escasez de estos fármacos, cobra vital importancia protocolizar el uso de otras alternativas farmacológicas, menos familiarizadas entre los equipos de salud. Esto, proporcionará las directrices necesarias para su utilización en forma óptima, estandarizada y segura. De allí que nuestro equipo propone, basado en recomendaciones recientemente publicadas ${ }^{30}$ y considerando las características farmacocinéticas y farmacodinámicas de los medicamentos disponibles, una serie de estrategias para el manejo de analgesia, sedación y bloqueo neuromuscular en pacientes críticos COVID-19, descritas en las Tablas 2 y 3. 


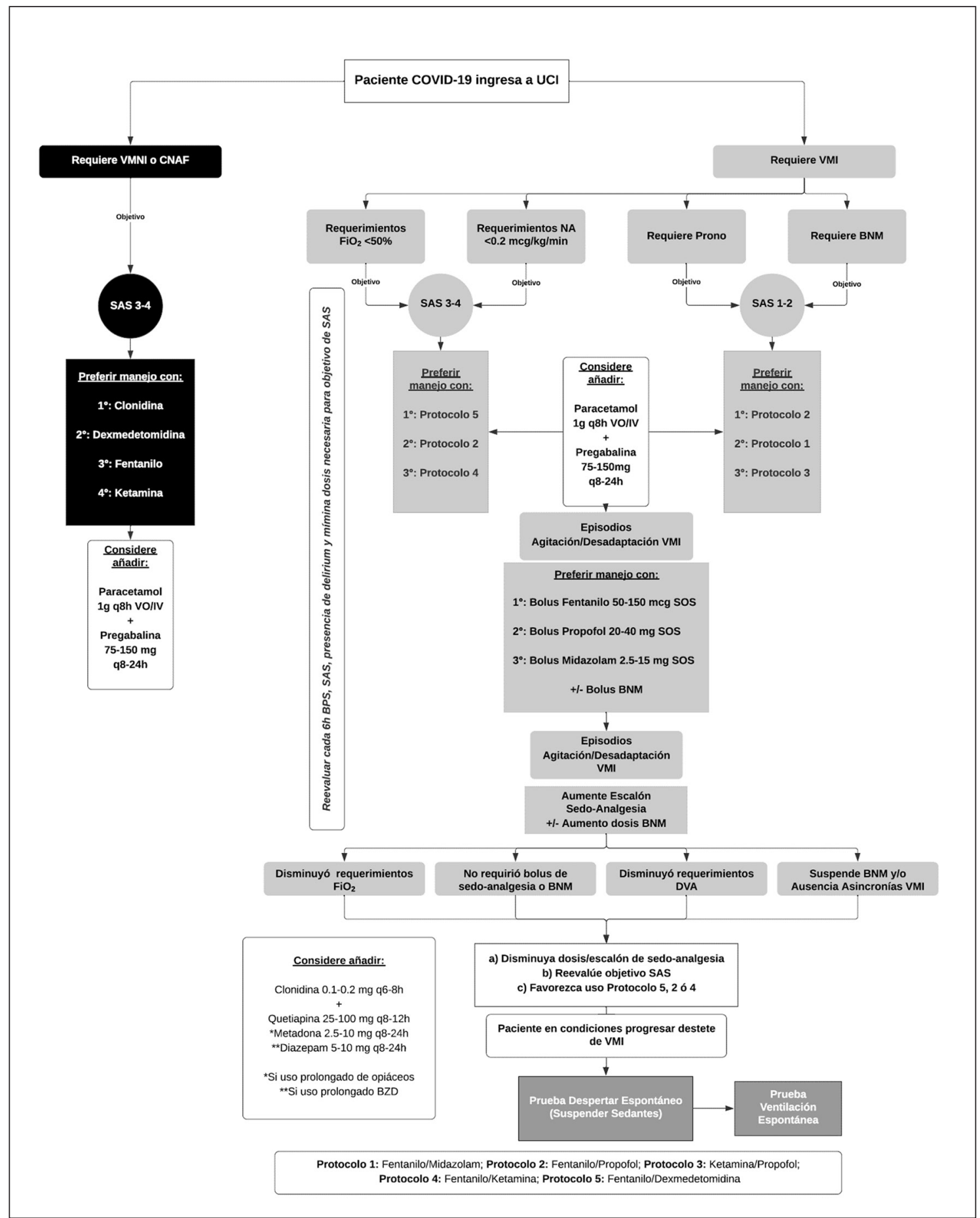

Figura 2. Abordaje protocolizado de sedación, analgesia y bloqueadores neuromusculares en el paciente crítico COVID-19. BNM: bloqueador neuromuscular; SAS: Riker Sedation-Agitation Scale; RASS: Richmond Agitation-Sedation Scale; AINEs: Antiinflamatorios no esteroidales; VMI: Ventilación Mecánica Invasiva; VMNI: Ventilación Mecánica no-Invasiva; CNAF: Cánula Nasal de Alto Flujo; Síndrome Distrés Respiratorio Agudo; $\mathrm{FiO}_{2}$ : Fracción inspirada de oxígeno; BZD: benzodiacepinas; VO: vía oral; IV: intravenosa. 


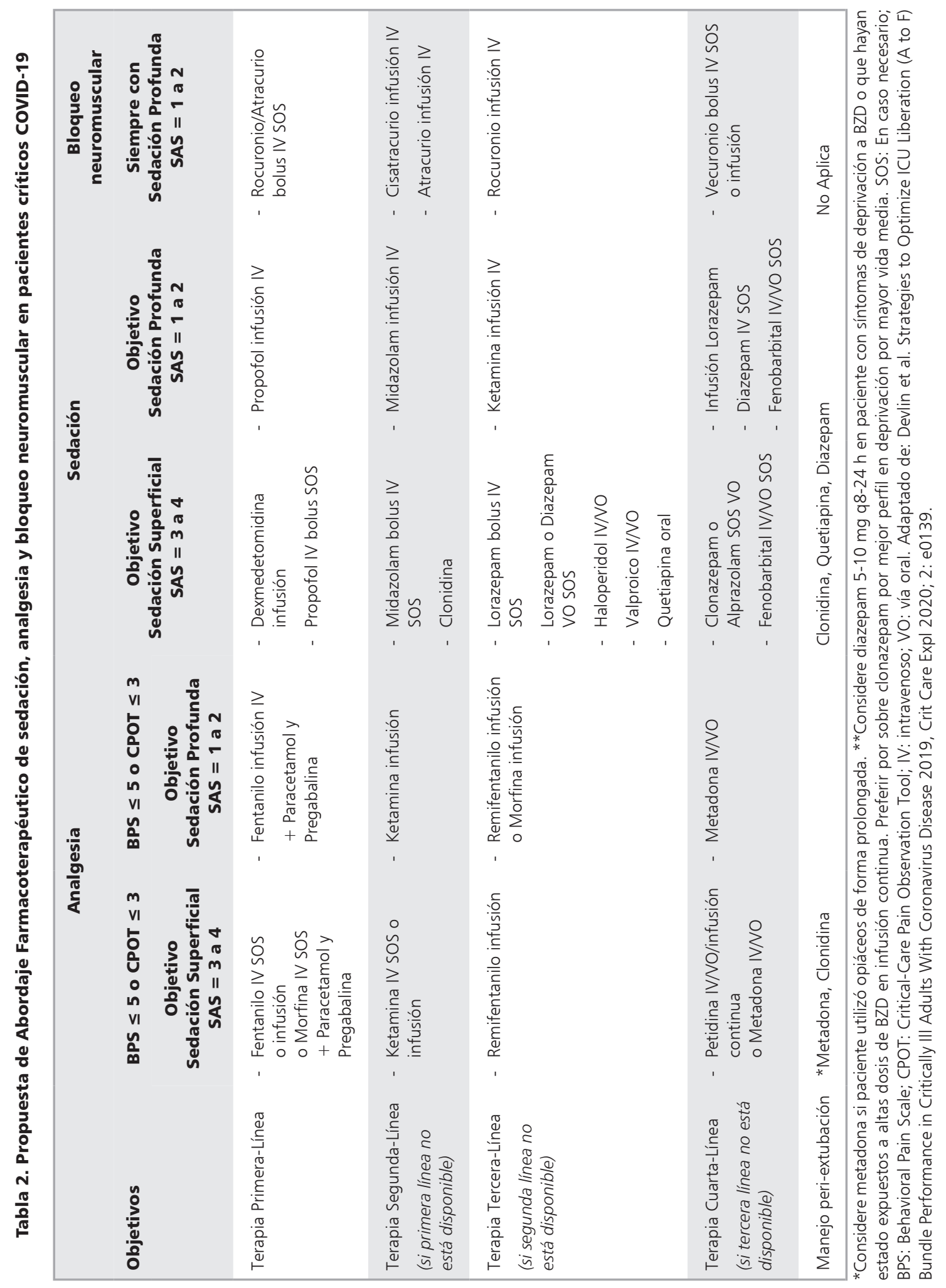




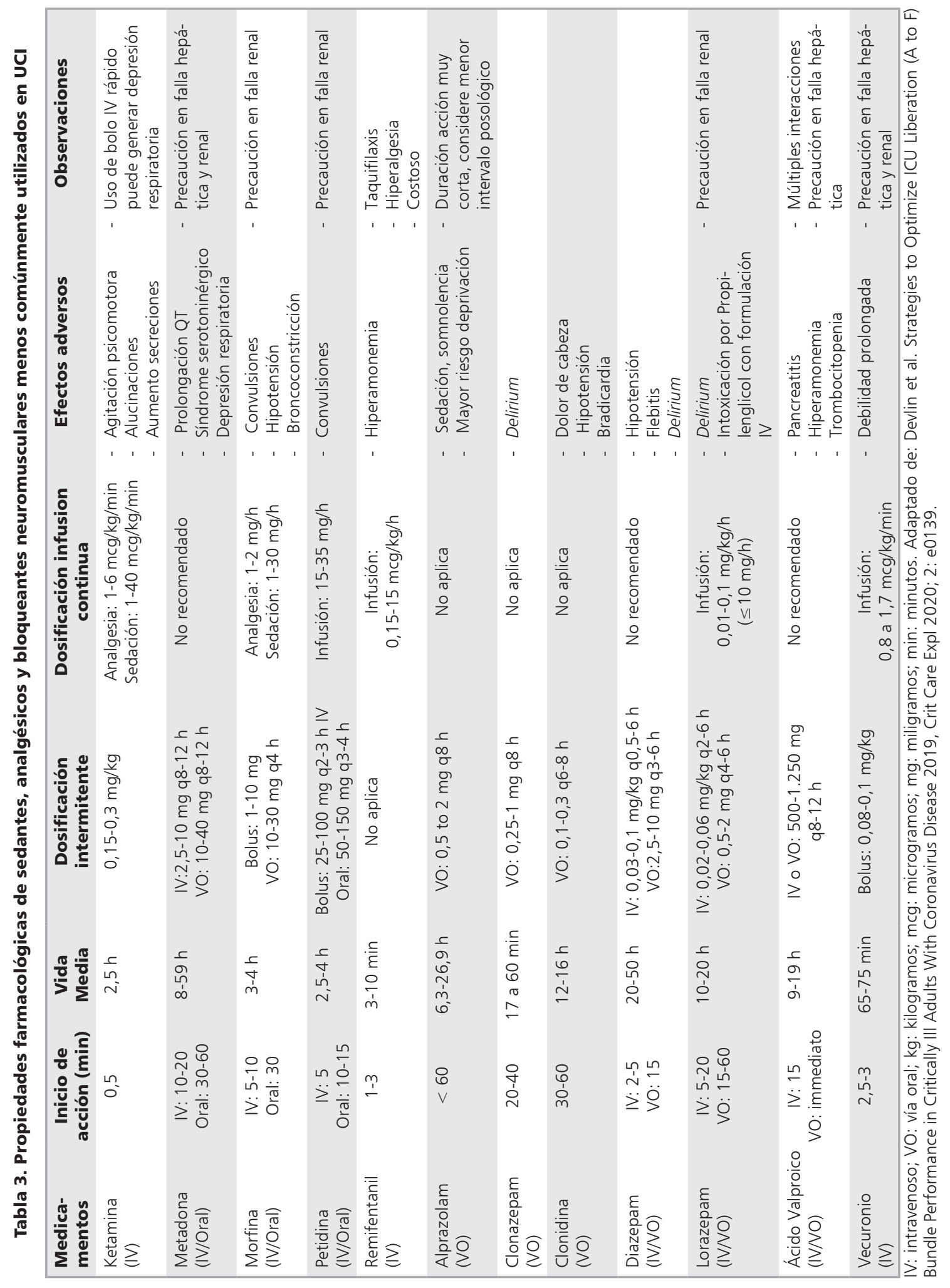




\section{Conclusiones}

Las consideraciones en la sedo-analgesia de los pacientes críticos con COVID-19 ha sido un desafío para los equipos de UCI. La optimización farmacológica mediante protocolos es considerado un elemento clave que debe ser considerado ante su impacto directo en los desenlaces clínicos, debiendo incluir alternativas terapéuticas de sedación ante períodos de escasez, con el fin de minimizar su impacto y facilitar su uso seguro y eficiente. Nuestra propuesta pretende aportar en el uso racional, costo-efectivo y con nivel aceptable de evidencia, de las opciones de sedación, analgesua y bloqueo neuromuscular en situación de pandemia, y no sustituye en ningún caso al juicio clínico que resulta de la valoración individual de cada paciente.

\section{Referencias}

1. Wu Z, McGoogan JM. Characteristics of and Important Lessons From the Coronavirus Disease 2019 (COVID-19) Outbreak in China: Summary of a Report of 72314 Cases From the Chinese Center for Disease Control and Prevention. JAMA. 2020; 323 (13): 1239-42.

2. World Health Organization. Coronavirus disease (COVID-2019) Situation Report -147. Disponible en: https:/www.who.int/docs/default-source/coronaviruse/situation-reports/20200615-covid-19-sitrep-147. pdf?sfvrsn=2497a605_4 [Consultado el 15 de junio de 2020].

3. Ministerio de Salud. Reportes Diarios Coronavirus 15 de junio, Enfermedad por SARS-CoV-2 (Covid-19) en Chile (2020 junio 15). Disponible en: https://cdn.digital. gob.cl/public_files/Campa\%C3\%B1as/Corona-Virus/ Reportes/15.06.2020_Reporte_Covid19.pdf [Consultado el 15 de junio de 2020].

4. Yang X, Yu Y, Xu J, et al: Clinical course and outcomes of critically ill patients with SARS-CoV-pneumonia in Wuhan, China: A singlecentered, retrospective, observational study. Lancet Respir Med. 2020; 8: 475-81.

5. Grasselli G, Zangrillo A, Zandella A, et al: Baseline characteristics and outcomes of 1591 patients infected with SARS COV-2 admitted to ICUs of the Lombardy region of Italy. JAMA. 2020; 323: 1574-81.

6. Bhatraju PK, Ghassemieh BJ, Nichols M, et al. Covid-19 in Critically Ill Patients in the Seattle Region - Case Series. N Engl J Med. 2020; 382 (21): 2012-22.

7. Olivares F, Muñoz D, Fica A, Delama I, Álvarez I, Nava- rrete M, et al. COVID-19 in Chile. The experience of a Regional reference Center. Preliminary report. MedRxiv 2020.06.14.20130898 [Versión no-publicada].

8. Muñoz Pichuante D, Villa-Zapata L. Benefit of Incorporating Clinical Pharmacists in an Adult Intensive Care Unit: A Cost-saving Study. J Clin Pharm Ther. 2020; 00: 1-7.

9. Lee H, Ryu K, Sohn Y, Kim J, Suh GY, Kim E. Impact on Patient Outcomes of Pharmacist Participation in Multidisciplinary Critical Care Teams: A Systematic Review and Meta-Analysis. Crit Care Med. 2019; 47 (9): 1243-50.

10. Jin Y-H, Cai L, Cheng Z-S, Cheng H, Deng T, Fan Y-P, et al. A rapid advice guideline for the diagnosis and treatment of 2019 novel coronavirus (2019-nCoV) infected pneumonia (standard version). Mil Med Res. 2020 Feb; 7 (1): 4.

11. Alhazzani W, Møller MH, Arabi YM, Loeb M, Gong MN, Fan E, et al. Surviving Sepsis Campaign: guidelines on the management of critically ill adults with Coronavirus Disease 2019 (COVID-19). Intensive Care Med. 2020.

12. Organización Panamericana de la Salud/Organización Mundial de la Salud. Guía para el Cuidado Crítico de Pacientes Adultos Graves con Coronavirus (COVID-19) En Las Américas. 2020; 1-67.

13. Scaravilli V, Grasselli G, Castagna L, et al. Prone positioning improves oxygenation in spontaneously breathing nonintubated patients with hypoxemic acute respiratory failure: A retrospective study. J Crit Care. 2015; 30 (6): 1390-4.

14. Sun Q, Qiu H, Huang M, Yang Y. Lower mortality of COVID-19 by early recognition and intervention: experience from Jiangsu Province. Ann Intensive Care. 2020; 10 (1): 33. Published 2020 Mar 18. doi:10.1186/ s13613-020-00650-2.

15. Ziehr DR, Alladina J, Petri CR, et al. Respiratory Pathophysiology of Mechanically Ventilated Patients with COVID-19: A Cohort Study. Am J Respir Crit Care Med. 2020; 201 (12): 1560-4. doi:10.1164/rccm.202004-1163LE.

16. Breen D, Karabinis A, Malbrain M, et al. Decreased duration of mechanical ventilation when comparing analgesia-based sedation using remifentanil with standard hypnotic-based sedation for up to 10 days in intensive care unit patients: a randomised trial. Crit Care 2005; 9: R200-10.

17. Rozendaal FW, Spronk PE, Snellen FF, et al. Remifentanil propofol analgo-sedation shortens duration of ventilation and length of ICU stay compared to a conventional regimen: a centre randomised, cross-over, 
open-label study in the Netherlands. Intensive Care Med. 2009; 35: 291-8.

18. Shehabi Y, Chan L, Kadiman S, et al. Sedation depth and long-term mortality in mechanically ventilated critically ill adults: a prospective longitudinal multicentre cohort study. Intensive Care Med. 2013; 39: 910-8.

19. Hanidziar D, Bittner E. Sedation of mechanically ventilated COVID-19 patients: challenges and special considerations. Anesth Analg. 2020 Apr 15:10.1213/ ANE.0000000000004887.

20. Huang Z, Chen YS, Yang ZL, Liu JY. Dexmedetomidine versus midazolam for the sedation of patients with non-invasive ventilation failure. Intern Med. 2012; 51 (17): 2299-305.

21. Senoglu N, Oksuz H, Dogan Z, Yildiz H, Demirkiran H, Ekerbicer H. Sedation during noninvasive mechanical ventilation with dexmedetomidine or midazolam: A randomized, double-blind, prospective study. Curr Ther Res Clin Exp. 2010; 71 (3): 141-53.

22. Ahmad I, Rathore FA. Neurological manifestations and complications of COVID-19: A literature review [published online ahead of print, 2020 May 6]. J Clin Neurosci. 2020;S0967-5868(20)31078-X.

23. Dixon M. Assessment and management of older patients with delirium in acute settings. Nurs Older People. 2018; 30 (4): 35-42.

24. Kotfis K, Williams Roberson S, Wilson JE, Dabrowski W, Pun BT, Ely EW. COVID-19: ICU delirium management during SARS-CoV-2 pandemic. Crit Care. 2020; 24 (1): 176. Published 2020 Apr 28. doi:10.1186/ s13054-020-02882-x

25. Girard TD, Pandharipande PP, Ely EW. Delirium in the intensive care unit. Crit Care. 2008;12 Suppl 3(Suppl 3): S3.

26. Kotfis K, Marra A, Ely EW. ICU delirium - a diagnostic and therapeutic challenge in the intensive care unit. Anaesthesiol Intensive Ther. 2018; 50 (2): 160-7.

27. Park SY, Lee HB. Prevention and management of delirium in critically ill adult patients in the intensive care unit: a review based on the 2018 PADIS guidelines. Acute Crit Care. 2019; 34 (2): 117-25.
28. Tobar E, Rojas V, Álvarez E, Romero C, Sepúlveda I, Cariqueo M, et al. Recomendaciones de la Sociedad Chilena de Medicina Intensiva para la analgesia, sedación, delírium y bloqueo neuromuscular en pacientes críticos médico-quirúrgicos adultos. Rev Ch Med Int. 2019; 34 (3): 1-29.

29. Morandi A, Piva S, Ely EW, et al: Worldwide survey of the "Assessing Pain, Both Spontaneous Awakening and Breathing Trials, Choice of Drugs, Delirium Monitoring/Management, Early Exercise/Mobility, and Family Empowerment" (ABCDEF) bundle. Crit Care Med 2017; 45: e1111-e1122.

30. Devlin J, O’Neal H, Thomas C, Barnes D, Mary A, Stollings J, et al. Strategies to Optimize ICU Liberation (A to F) Bundle Performance in Critically Ill Adults With Coronavirus Disease 2019, Crit Care Expl. 2020; 2: e0139.

31. Gupta M, Gokarakonda SB, Attia FN. Withdrawal Syndromes. [Actualizado 2020 May 26]. In: StatPearls [Internet]. Treasure Island (FL): StatPearls Publishing; 2020 Jan. [Disponible en: https://www.ncbi.nlm.nih. gov/books/NBK459239/].

32. Duby JJ, Berry AJ, Ghayyem P, Wilson MD, Cocanour CS. Alcohol withdrawal syndrome in critically ill patients: protocolized versus nonprotocolized management. J Trauma Acute Care Surg. 2014; 77 (6): 938-43.

33. Zhang W, Zhao Y, Zhang F, et al. The use of anti-inflammatory drugs in the treatment of people with severe coronavirus disease 2019 (COVID-19): The Perspectives of clinical immunologists from China. Clin Immunol. 2020; 214: 108393.

34. Papazian L, Forel JM, Gacouin A, et al. Neuromuscular blockers in early acute respiratory distress syndrome. N Engl J Med. 2010; 363 (12): 1107-16.

35. The National Heart, Lung, and Blood Institute PETAL Clinical Trials Network, Moss M, et al. Early neuromuscular blockade in the acute respiratory distress syndrome. N Engl J Med. 2019; 380 (21): 1997-2008.

36. Bass S, Vance ML, Reddy A, et al. Bispectral index for titrating sedation in ARDS patients during neuromuscular blockade. Am J Crit Care. 2019; 28 (5): 377-84. 